\title{
UNA PANORÁMICA SOBRE LA RESTRICCIÓN CONSERVADORA DE LA ESTRUCTURA ${ }^{1}$
}

\author{
Víctor M. Longa* \\ Universidad de Santiago de Compostela
}

\begin{abstract}
RESUMEN: El desarrollo de la GGT consiste sobre todo en intentar aunar las necesidades descriptivas y las explicativas. Ésta es precisamente la principal causa por la que se han producido modificaciones en los diferentes modelos propuestos. En este artículo se incide en una de las restricciones que más han ayudado a esa labor, la Restricción Conservadora de la Estructura (RCE). Sin embargo, en su formulación original esta restricción estaba bastante limitada debido a ciertas inadecuaciones, y a su carácter no general (se refería únicamente al componente transformacional). En el presente trabajo se da cuenta de las mismas, y del modo en que fueron superadas, y también se pone de manifiesto cómo la RCE pudo extenderse a todos los componentes y niveles, haciéndola verdaderamente rentable para la teoría de la gramática.

ABSTRACT: The development of Generative Grammar has been basically aimed to unite its descriptive and explicative necessities. This is the main motivation underlying the modifications of its different versions. This paper focuses on one of the constraints that has helped most to achieve this task, namely Emonds' (1970, 1976) Structure-Preserving Constraint (SPC). It must be said, however, that this constraint was rather limited in its original formulation because of some inconsistencies and of its restricted nature (it was only referred to the transformational component). This article outlines the aforementioned inconsistencies and explains how they have been overcome and how the SPC has been extended to other components and levels making it a profitable constraint for the theory of grammar.
\end{abstract}

\section{Introducción}

El desarrollo de la gramática generativa transformacional (GGT) constituye en gran medida el intento de resolver la tensión entre los requisitos descriptivos y los explicativos. Chomsky (1986:52) escribe al respecto unas palabras muy significativas:

* Profesor Ayudante de Lingüística General, Dpto. de Filoloxia Española, Teoría da Literatura e Lingüística Xeral.

1. Deseo agradecer muy vivamente los comentarios de Guillermo Lorenzo (Universidad de Oviedo) a una versión previa de este trabajo. 
To achieve descriptive adequacy, it often seems necessary to enrich the system of available devices, whereas to solve our case of Plato's problem we must restrict the system of available devices so that only a few languages, or just one, are determined by the given data.

Tras percibir pronto que el modelo de Chomsky (1965) no podía solucionar satisfactoriamente el enfrentamiento entre descripción y explicación, los generativistas vieron muy clara la necesidad de limitar el número y poder de las reglas, de modo que poco a poco se fueron eliminando éstas en favor de principios muy generales y abarcadores, hasta llegar al modelo 'Government \& Binding' (GB; cfr. Chomsky, 1981). Algunos desarrollos importantes que permitieron tal paso fueron, por ejemplo, la eliminación del orden extrínseco de las reglas en favor de uno intrínseco ${ }^{2}$, la extensión del principio de la aplicación ciega de las transformaciones ${ }^{3}$, o la sintaxis Xbarra, que redujo hasta el mínimo el aparato sintagmático.

La restricción a la que alude este trabajo constituye otra de esas propuestas centrales, hasta el punto de que constituye una de las reducciones del mecanismo expresivo más fructíferas de las formuladas en la GGT, debido a su repercusión: por esta razón constituye una de las restricciones que más han ayudado a resolver la tensión antes aludida, teniendo en cuenta además que, desde su postulación, no ha sido abandonada en ningún momento posteriormente, sino extendida como condición operante sobre toda la gramática. Me refiero a la Restricción de la Conservación de la Estructura [Structure-Preserving Constraint; en adelante, RCE], formulada en Emonds (1970) y (1976) ${ }^{4}$.

En las siguientes páginas me propongo, en primer lugar, señalar las causas generales que motivan la postulación de la mencionada restricción. En la sección 3 se presentará la formulación de la RCE, la cual tenía algunas deficiencias que debieron ser pulidas posteriormente para hacerla verdaderamente rentable: en la 4 se dará cuenta de las mismas. En la 5 se abordará el modo tan satisfactorio en que las deficiencias fueron solventadas, extendiendo la RCE a toda la teoría gramátical, mientras que en la última se mencionará la causa por la que se ha podido acceder a una generalización de la preservación estructural.

Deseo precisar que este trabajo no pretende ser, en modo alguno, una crítica a la RCE, puesto que ésta ha resultado decisiva en la GGT desde su formulación hasta la actualidad, sino que pretende constituir un ejemplo del modo de proceder en GGT, reinterpretando y extendiendo ideas, de manera que sirvan para posteriores revisiones

2. En gran medida motivada por la extensión de la Condición del Ciclo Estricto, formulado en Chomsky (1965:134-5), pero no verdaderamente operativo hasta el marco de Chomsky (1973).

3. Efectuada en Chomsky (1976:312), pero igualmente presente en Chomsky (1965:142-3).

4. Sin embargo, tal importancia no se ha visto reconocida en la práctica, como señala Newmeyer (1980:251). 
de la teoría, al tiempo que, más específicamente, pretende incidir en la cada vez mayor importancia de la RCE.

\section{Contexto del surgimiento de la RCE.}

Es bien conocido que el modelo clásico, Chomsky (1965), se basaba en reglas, de varios tipos: algunas de ellas generaban la estructura profunda, mientras que otras variaban tal nivel hasta obtener la estructura superficial correspondiente; posteriormente, el resultado era ajustado mediante reglas morfofonológicas.

Ciñéndonos al subcomponente transformacional, éste se componía de operaciones bastante diferentes en cuanto a su naturaleza, cuyo único nexo de unión consistía en que efectuaban cambios sobre la estructura profunda (o sobre su derivación). Así, las transformaciones obedecían a diversas gamas operacionales:

(i) Inserción: consiste en añadir un elemento, inexistente hasta ese momento en la derivación, pero necesario para el buen curso de la misma, como la inserción de la partícula negativa, del complementante, de un elemento relativo, etc.

(ii) Sustitución: un elemento presente en la derivación debe ser cambiado por otro inexistente en ella, como la supresión de FN en favor de la introducción de un clítico.

(iii) Movimiento: un constituyente presente en la etapa $\mathrm{X}$ de la derivación debe ser desplazado a otra posición estructural (usualmente, creada con tal movimiento). Ejemplos clásico pueden ser la subida de clíticos o la pasivización.

(iv) Elisión: un elemento debe ser suprimido, sin que quede reminiscencia alguna de su anterior presencia en ella, como el borrado de la FN dominada de modo inmediato por el pronombre relativo para formar secuencias de relativo.

La conclusión de estas diferentes posibilidades es sencilla: las transformaciones pueden efectuar cualquier tipo de alteración con tal de que se asegure una correcta derivación hacia el nivel superficial. Pero, además, dentro de cada gama se formulaban reglas muy variadas, puesto que dependiendo de las necesidades concretas de la derivación, se postulaban múltiples modos de efectuar una inserción o un movimiento, por ejemplo. Esto es, se podían (en realidad, se debían) formular reglas diferentes para construcciones diferentes.

Esta situación estaba motivada por el poder tan grande de las reglas en el modelo clásico. Chomsky (1965:24-6) formuló tres niveles de adecuación de las gramáticas, y de la teoría lingüística asociada: observacional, descriptivo y explicativo. El nivel más apetitoso, dados sus objetivos, es el explicativo, ya que pretende ofrecer una hipótesis de la adquisición del lenguaje por el niño. Si éste aprende con tanta facilidad

5. La tipología que a continuación se especifica es la usualmente reconocida; así se puede encontrar, por ejemplo, en D'Introno (1979:49,295). 
y rapidez una lengua, tal hipótesis debe ser severamente acotada, permitiendo un número muy pequeño de opciones entre las que el infante debe decidir en el proceso mencionado. Sin embargo, en la práctica, en el modelo estándar sólo se aborda la cuestión de la adecuación descriptiva: no se pasó de la descripción, como señala Kimball (1973:xvi): Perhaps the only generally shared point of agreement concerning the Chomskyan theory (Chomsky 1965) is that it is inadequate as a theory of universal grammar. [paréntesis: VML] ${ }^{6}$

Precisamente, aquí nos hallamos ante la tensión entre descripción y explicación a la que antes aludí. La adecuación descriptiva exigía unos mecanismos muy ricos y exhaustivos, pero al tiempo, tales mecanismos impedían logros en el terreno de la adecuación explicativa. Al estar basada la gramática en reglas, el poder expresivo de la teoría estaba en situación directamente proporcional a la adecuación descriptiva: cuanto más detalladas fueran las descripciones de las secuencias, más se avanzaba en la descripción; pero tal poder estaba en relación inversamente proporcional a los logros explicativos. La razón es que las reglas se limitan a traducir a términos formales las secuencias generadas, de modo que al depender todo el modelo de reglas, el formalismo no podía generalizar más allá de la propia generalización existente en las estructuras explicitadas.

Llevando esto al terreno de las transformaciones, podremos intuir el por qué de la facilidad con la que las transformaciones podían efectuar cualquier suerte de alteración sobre las derivaciones ${ }^{7}$, e igualmente, el por qué del excesivo poder de tales reglas.

Esto es, de los dos objetivos centrales, dar cuenta de la competencia y ofrecer una hipótesis sobre la carga innata que facilita la adquisición ${ }^{8}$, sólo se podía abordar el primero, pero no el segundo, por lo mencionado.

Notemos además que, en una teoría tan preocupada por el correlato psicológico, la excesiva proliferación de las reglas en general y de las transformaciones en particular se presentaba como un hecho nada adecuado en relación precisamente a los requisitos que tal realidad psicológica precisaba. Era un contrasentido intentar explicar la

6. Parecidas afirmaciones se pueden hallar en D'Introno (1985:29) o Wasow (1985:195, n. 4).

7. Sería injusto negar la presencia explícita de alguna restricción sobre tales reglas, como fundamentalmente, la enunciada en Katz \& Postal (1964:47,66), relativa a que las transformaciones no podían alterar el significado básico subyacente a lo largo de la derivación (se asumía que sólo el nivel de la estructura profunda era relevante para la interpretación semántica). Una consecuencia de ello consistía en que si alguna regla producía una elisión, el elemento borrado debía ser recuperable. Sin embargo, tal restricción no gozaba de poder operativo real, por varias razones: por un lado, Peters \& Ritchie (1973) demuestran formalmente que la posibilidad de recuperar lo borrado es irreal en bastantes ocasiones. Por otro lado, reglas como la de pasividad cambiaban claramente el significado subyacente. Además, en Chomsky (1965:142-3) se formula que las transformaciones deben ser insensibles al contexto, pero si ello es así, sería bien difícil recuperar un elemento sin tener en cuenta precisamente tal contexto. Desde todos los puntos de vista, la condición enunciada por Katz \& Postal (1964) se revela como inoperativa.

8. En el sentido especificado en Chomsky (1986:41-2): estado estable e inicial respectivamente. 
rapidez con que un infante adquiere el lenguaje mediante un modelo que necesitaba una ingente cantidad de operaciones, muy específicas, para dar cuenta de esa rapidez; el niño necesitaría saber qué regla concreta precisa, y en cuál de las gamas se integra. Así, explicar tal rapidez mediante el tan recargado sistema transformacional ayudaba a todo menos a explicar esa rapidez ${ }^{9}$.

De todos modos, ya en su (1965), Chomsky es consciente de la limitación mencionada, puesto que en varias ocasiones subraya la necesidad de restringir el número y la clase de las gramáticas accesibles (ibid.: 27-8,35,46). Así, los excesivos mecanismos del modelo se asumían como un defecto implícito al formalismo. Para demostrar formalmente tal defecto, fueron muy importantes Peters \& Ritchie (1971) y (1973), que demuestran lo hasta entonces intuido: el excesivo poder del modelo, motivado por una capacidad generativa débil y fuerte demasiado potentes de cualquier gramática que proveyera el modelo clásico. Dicho en pocas palabras, el modelo podía generar más secuencias que las gramaticales, y por tanto, sobregeneraba (capacidad generativa débil), y también generaba en exceso al ofrecer descripciones estructurales sobre las generaciones, puesto que producía descripciones alternativas para los mismos hechos (capacidad generativa fuerte) entre las que la teoría no podía optar ${ }^{10}$, debido a la oscilante relación entre reglas sintagmáticas y transformaciones. Del mismo modo en que los resultados del componente sintagmático están carentes de contenido (cfr. Peters \& Ritchie 1971), también lo está el sistema transformatorio (cfr. Peters \& Ritchie 1973). Por ello, el modelo no afirma nada sobre las lenguas, salvo que éstas pueden ser generadas mediante un conjunto de reglas. Y, en relación a la adecuación explicativa, lo único que se afirma es que hay reglas sintagmáticas y transformacionales, pero este contenido se pierde debido al gran número de reglas necesitadas.

Así, el principal objetivo de Chomsky (1965), aislar unas pocas opciones de gramáticas accesibles entre las que debiera decidir el niño, se quedaba en mero objetivo, puesto que existía una gran cantidad de posibilidades. Por ello, según Chomsky (1972:70) era necesaria

a linguistic theory that has the latitude to meet requirements of descriptive adequacy, but is sufficiently complex, highly articulated, and specific in its constraints so that the problem of explanatory adequacy can at least be placed on the agenda.

9. Cfr. Berwick \& Weinberg (1986) y Weinberg (1988) como trabajos en los que se aborda esta cuestión, en relación sobre todo a las reglas transformacionales.

10. Cfr. sobre ambas nociones Chomsky (1965:60). Es clásico en relación a esta capacidad el debate del orden de palabras en alemán, entre Bach (1962) y Ross (1970) (trabajo que data de 1967), en donde se da cuenta de idénticos datos de dos modos alternativos permitidos por el marco de la teoría de la gramática, por lo que ésta se ve imposibilitada para decidir entre ambos. 
Tal necesidad de reducir las reglas transformacionales (al igual que el resto) condujo al desarrollo de un programa de investigación que intentó controlarlas, cada vez más fructíferamente desde el punto de vista explicativo. Dicha reducción transformacional se efectuó en dos direcciones diferentes pero a la vez conectadas, que acabarían por confluir:

1. En primer lugar se intentó reducir la multiplicidad de reglas en el dominio de la extracción de elementos, proponiendo generalizaciones que subsumieran diferentes formulaciones particulares en una sola regla, más global; de este modo, también se podían generalizar tanto las definiciones estructurales como los cambios a realizar sobre la estructura. En esta línea se inscriben el Principio de A-sobre-A (Chomsky 1964:930-1), las restricciones de Ross (1967) y las condiciones de Chomsky (1973). Sin embargo, esta vía no atacaba de raíz el problema de la riqueza excesiva de mecanismos, porque se refería únicamente a las operaciones de movimiento, sin abordar las otras tres operaciones que las transformaciones podían realizar.

2. Por ello, la segunda aproximación comenzó a incidir en la reducción de la propia tipología operacional antes señalada, de forma que paulatinamente pudieran ser eliminadas todas aquellas reglas que no se limitaran a desplazar un constituyente. Es en esta segunda vía donde se puede situar la RCE, y precisamente su importancia está motivada por el hecho de que constituye el inicio de este segundo intento de acotar los mecanismos gramaticales.

\section{La RCE.}

En Kimball (1972) se defiende que los resultados de las transformaciones cíclicas equivalen a los generados por las reglas sintagmáticas ${ }^{11}$. Esta idea, retomada en Emonds (1970) y (1976), e integrada en un marco más amplio, tendría unas decisivas consecuencias en relación a la segunda vía citada de reducción de las transformaciones.

Emonds, como todos los generativistas en aquellos tiempos, reconoce el gran problema de la indecuación del componente transformacional, de su excesivo poder generativo; tal como señala (1976:vii), "For those working in generative syntactic theory, it has long been recognized that any formally manageable and empirically significant generative model must drastically reduce the expressive power of grammatical transformations".

La pretensión de Emonds consiste en intentar reducir ese excesivo poder de las reglas (de una parte de ellas), tomando precisamente la idea de Kimball como uno de los factores principales para limitar ciertas reglas. Emonds tiene en cuenta si los resultados que producen ciertas transformaciones se ajustan estrictamente a los alcanzables

11. Si bien Newmeyer (1980:249) señala que tal idea data de 1967. De ahí que el aparente anacronismo con respecto a Emonds (1970) no sea tal. 
por las reglas sintagmáticas; en el caso de que existan desfases entre ambos resultados, las transformaciones formuladas deben rechazarse. Por ello, tal necesidad comprobatoria es en principio poderosa, porque pretende justificar de modo independiente los resultados de las transformaciones; si de manera ajena a ellas se confirman sus resultados, las reglas quedan legitimadas, puesto que conservan la estructura que podría generarse mediante reglas sintagmáticas.

Sin embargo, no todas las transformaciones pueden someterse a la referida restricción; en opinión de Emonds (1976:3-4), existen 3 tipos de transformaciones:

1. Radicales [Root]: en ellas, un elemento queda dominado por un nudo O(ración) que no depende de ningún otro nudo O. En resumidas cuentas, son las aplicadas en las secuencias matrices. Ejemplos de ellas son reglas como tematización, inversión sujeto-auxiliar, etc.

2. Locales [Local]: aplicadas sobre constituyentes estrictamente adyacentes, de modo que la regla no está sujeta a ninguna condición exterior al dominio estricto en el que ambos constituyentes residen, un nudo O. Son operaciones como movimiento del artículo, etc.

El tercer tipo es definido con las propias palabras de Emonds (1976:3):

Structure-Preserving Transformation: A transformation (or a transformational operation, in the case of a transformation performing several operations) that introduces or substitutes a constituent $\mathrm{C}$ into a position in a phrase marker held by a node $\mathrm{C}$ is called "structure-preserving"12. [negritas: JEE; nota: VML]

Así pues, una regla preservadora de la estructura mueve un elemento a una posición previamente existente, en la que tal elemento y su posición pueden ser generados de modo independiente por el componente sintagmático, legitimando la operación. Además, el constituyente debe moverse a una posición compatible con su naturaleza categorial. Si se atiene a estos requisitos, la regla satisfará la RCE, definida en Emonds (1976:5):

12. En relación al término 'substitution', que aparece en la definición de Emonds, debe matizarse algo importante; en el apartado anterior se han mencionado los 4 tipos principales de operaciones transformatorias; una de ellas era la sustitución, que borraba un elemento insertando otro en su lugar. Sin embargo, no es éste el sentido aludido por el término de Emonds, puesto que en él se refiere a una operación que no permite realizar modificaciones con elementos que no estén presentes en la etapa concreta de la historia transformacional. Como veremos, tal sustitución se produce mediante el desplazamiento de un constituyente a una posición que ya existía previamente, aunque vacía de contenido léxico. Por esta razón, la sustitución respeta la estructura, puesto que no se crea ni destruye ninguna posición. De hecho, en la obra que oficialmente inaugura el modelo GB, Chomsky (1981), se menciona la sustitución y la adjunción como subtipos del desplazamiento de constituyentes (única operación legitimada). Es precisamente a este sentido al que alude Emonds (por supuesto, de manera bastante incipiente), y no al primeramente señalado. 
Structure-Preserving Constraint: Major grammatical transformations are either root or structure-preserving operations. [negritas: JEE]

Un caso paradigmático de regla en la que actúa la RCE es la de pasivización. Siguiendo a Chomsky (1970) y (1973), Emonds subdivide la regla clásica de pasivización en dos diferentes; consideremos (secuencias tomadas de Emonds, 1976:66):

\section{(1) Russia defeated Germany}

(2) Germany was defeated by Russia

Para obtener (2) a partir de (1) son necesarios dos desplazamientos: mediante el primero, la FN 'Russia' de la estructura profunda (1) (en la que se han omitido todas las especificaciones propias de tal nivel) debe postponerse a la FN 'Germany', mientras que el segundo motiva que 'Germany' se anteponga a V. Los resultados de ambas reglas son confirmados por mecanismos independientes: la primera, postposición de FN, mueve este elemento a una posición en la que queda subsumida por la FN encabezada por 'by'. Tal resultado es confirmado por el componente sintagmático, que puede generar una secuencia como FP $\rightarrow$ P FN, justificando esta generación el resultado de la transformación. En cuanto a la segunda regla, anteposición de FN, de nuevo el componente de la base confirma la legitimidad del resultado: una regla sintagmática producirá la estructura $\mathrm{O} \rightarrow \mathrm{FN}$ Aux FV. De este modo, ninguno de los dos movimientos atenta contra las estructuras generables por la base.

Sin embargo, aunque necesaria, esa premisa no es suficiente para que la derivación transformacional no altere la estructura, esto es, no cree posiciones nuevas ni elida otras ya presentes. Emonds postula que ambos desplazamientos no atentan contra la imposibilidad de crear posiciones nuevas gracias a la existencia de unos nudos vacíos presentes ya en la estructura profunda, que representa mediante el símbolo ' $\triangle$ '; tales nudos pueden recibir un constituyente que se haya desplazado, saturando tal posición ${ }^{13}$. Siguiendo a Chomsky (1972:121, n. 12), Emonds señala que tales nudos deben haber desaparecido una vez que la derivación ha conformado la estructura superficial, puesto que en caso contrario, la misma devendrá agramatical. Esto significa que, en el curso de la historia transformacional, tales nudos deben haber sido llenados por elementos léxicos. Por ello, no es necesario crear ninguna nueva posición, ni perder tampoco ninguna otra; en resumen, se conserva la identidad estructural en todos los pasos de la derivación. Retomando (1), que repito aquí

13. Aunque en este momento la noción de 'huella' no estaba extendida, debe destacarse que diversos tipos de elementos vacíos ya habían sido usados anteriormente, como p. ej., 'doom markers' empleados por Ross (1969) y Postal (1970). Sin embargo, estos elementos vacíos no gozaban de la sistematicidad de la que dispondrían cuando se generalizara la teoría de la huella, y por otro lado, su empleo solía ser ad hoc en bastantes ocasiones, para asegurar la aplicación de reglas posteriores. 
(1) Russia defeated Germany

su estructura previa al primer movimiento es como sigue:

(3) $\mathrm{O}\left[\right.$ Russia $\mathrm{T}$ [ed] $\mathrm{FV}$ [defeat $\mathrm{FN}\left[\right.$ Germany $\left.\left.\left.\mathrm{FP}^{[\text {by }} \mathrm{FN}[\triangle]\right]\right]\right]^{14}$

La FN más alta jerárquicamente se traslada a la posición $\triangle$ al cumplirse las exigencias requeridas para ello: la FN pertenece a la misma clase categorial de la posición vacía, y además, no es preciso estipular ninguna posición nueva en el curso de la derivación. Pero puesto que esa FN se desplaza de su posición primitiva, su partida crea a su vez un nuevo elemento $\triangle$ en tal posición; la estructura resultante se representa en (4):

\section{(4) $\mathrm{O}[\mathrm{FN}[\triangle] \quad \mathrm{T}$ [ed] $\mathrm{FV}$ [defeat $\mathrm{FN}[$ Germany] $\mathrm{FP}$ [by $\mathrm{FN}$ [Russia]]]]}

La posición vacía presente en (4) permite a la FN 'Germany' moverse a ese lugar y ocuparlo, con lo que el resultado es ya (2), repetido de nuevo:

(2) Germany was defeated by Russia 15

Recapitulando, se puede apreciar que las diferentes operaciones, elementos y posiciones implicadas en la derivación satisfacen los imperativos de la RCE, porque:

1. Las posiciones y constituyentes usados han sido exactamente los mismos en todas las etapas de la derivación: no se ha necesitado elidir ni introducir ninguna/o.

2. En ambos movimientos un elemento dotado de los rasgos categoriales [+N,$\mathrm{V}$ ], una FN, se traslada a una posición receptora que coincide en esos mismos rasgos, corroborando así la siguiente imposición de Emonds (1976:68): “A transformational operation $\mathrm{T}$ that substitutes a node $\mathrm{B}$ and all the material dominated by it for some node $\mathrm{C}$ that is a constituent of the same category is structure-preserving."

14. Emonds (1976:69) propone dos tratamientos de la FN agente, que asumen ambos su presencia ya en la estructura profunda: (i) ‘by’ ya está presente como cabeza de una FN vacía, o (ii) ‘by’ es insertado en una FP vacía.

15. Con el análisis postulado, Emonds resuelve al tiempo el problema de la opcionalidad del agente, hasta entonces generado en la estructura profunda, siendo elidido posteriormente si no se reflejaba en la estructura superficial. Sin embargo, una elisión es incompatible con el marco de este autor, puesto que destruiría estructura (una posición). Además, Emonds señala el carácter claramente ad hoc de tal elisión. Por ello, la opcionalidad de la FAgente puede atribuirse a la opcionalidad de la presencia de la misma en el nivel subyacente; si este nudo no aparece en ella, sólo se producirá un movimiento, el de la FN 'Germany', que ocupará la posición vacía. 
3. Además, los resultados de las dos reglas transformacionales son confirmados de modo independiente, ya que son justificados por reglas sintagmáticas, capaces de generar las mismas (sub)estructuras que las producidas por vía transformacional.

Por estas razones, se satisface la forma de una transformación preservadora de la estructura, que en opinión de Emonds (1976:69) es como sigue:

a. $\mathrm{W}-[\mathrm{B} X]-\mathrm{Y}[\mathrm{B} \triangle]-\mathrm{Z}$

b. $\mathrm{W}-[\mathrm{B} \triangle]-\mathrm{Y}-[\mathrm{B} \mathrm{X}]-\mathrm{Z}^{16}[$ nota: $\mathrm{VML}]$

Estos son, brevemente esbozados, los mecanismos implicados en las transformaciones preservadoras de la estructura. A la vista de ellos, se percibe que la formulación de Emonds pretende poner freno a las derivaciones transformacionales, usualmente bastante costosas.

\section{Algunos aspectos problemáticos de la RCE.}

Cuando señalé, en el final del apartado 2., las dos vías mediante las que se intentaron reducir los mecanismos transformacionales, mencioné que la RCE constituía el primer intento de reducir las diferentes gamas operacionales de las transformaciones. Por ello, esta restricción es muy importante, ya que sienta las bases de lo que supondrá la eliminación de la citada tipología. Pero también señalé que esta importancia lo es en espíritu más que en su letra, porque la RCE contiene varios aspectos problemáticos, que con el paso del tiempo se fueron limando, hasta que la conservación de la estructura resultó ser un verdadero principio constrictor no sólo sobre las reglas transformacionales sino sobre todo el modelo. A continuación, se pondrán de manifiesto y discutirán los aludidos aspectos problemáticos.

4.1. La RCE en realidad no puede limitar en su conjunto las historias transformacionales, sino que sólo desecha las generaciones incorrectas motivadas por la aplicación de reglas inadecuadas. Esto lo expresa bien Newmeyer (1980:251) cuando señala que la RCE excluye análisis posibles pero no limita la clase de los plausibles.

La mayor parte de los análisis que no usen una regla incorrecta (que no sea radical ni local) resultarán ser estructuralmente conservadores. En este sentido, la restricción más fuerte aducida por Emonds, la justificación del resultado de la transformación mediante la generación en la base, es mucho más permisiva de lo que parece, dado que

16. Notemos que en el segundo movimiento, 'Germany' se mueve a la posición inicial de la secuencia sin dejar esta vez un nudo vacío, supuesto que causaría la agramaticalidad de la misma (cfr. infra). Si sólo se considerara esa necesidad, la no estipulación de una posición vacía tras haberse movido esa FN podría verse como un hecho ad hoc. Sin embargo, ese posible resultado no podría obtenerse mediante las reglas sintagmáticas, que no podrían generar una posición vacía. 
el resultado de cualquier regla transformatoria que coincida con las posibilidades (tan amplias) ofrecidas por el componente sintagmático será correcto. Suponiendo que dos descripciones alternativas para unos mismos hechos coincidan con resultados de las reglas sintagmáticas, la RCE no podrá desechar una de ellas, por lo que no puede limitar el número de descripciones compatibles con los datos.

Si relacionamos lo mencionado con las conclusiones a las que llegaron en su examen de la teoría estándar Peters \& Ritchie (cfr. apdo. 2.), vemos que la RCE no sirve de mucho a la hora de pulir los defectos formales del modelo. Casi todos los análisis que no usen una regla incorrecta, o que no apliquen una regla de modo inadecuado, resultarán ser preservadores. En otras palabras, los análisis alternativos que lleguen a un mismo resultado, concordante con una generación sintagmática correcta, serán preservadores. Esto significa que la RCE no puede restringir el poder de la capacidad generativa fuerte; es éste el verdadero problema, pues una capacidad generativa fuerte por exceso significa un poder generativo (de descripciones, no de secuencias) demasiado fuerte. Y afirmar esto significa que automáticamente la RCE se integra en los mecanismos que potencian el mencionado poder.

Recordemos que Peters \& Ritchie (1973) achacaban buena parte de culpa del excesivo poder generativo del modelo a la nada clara relación entre reglas sintagmáticas y transformatorias; dependiendo de cuál de ellas resultara primada, los resultados descriptivos realizados sobre las secuencias podían variar grandemente. Emonds vincula la aplicación de las transformaciones preservadoras de la estructura a la confirmación de su resultado por medio de las reglas sintagmáticas, pero no consigue por medio de esta relación la resolución del problema señalado en Peters \& Ritchie (1973).

4.2. Otro problema importante que presenta la RCE es el siguiente: es evidente que si las reglas transformacionales (o algunas, en su defecto) deben preservar la estructura, muchas operaciones que inserten o supriman elementos deben ser abolidas, como así sucede de hecho. En este sentido, el deseo de Emonds de encontrar justificación independiente a los resultados de las transformaciones es muy interesante, puesto que de esta forma intenta anular lo que sin un refrendo autónomo se presentaría como una estipulación ad hoc, una carga aún más pesada para los mecanismos, esto es, para el poder expresivo, con lo que la restricción sería subsumida bajo estos factores mencionados. Sin embargo, si para ello confronta los resultados de la aplicación de las reglas transformacionales con los de las sintagmáticas, de modo que para que una de aquéllas quede legitimada deba producir el mismo resultado que una de éstas podría generar, la pregunta resulta obvia y nada trivial: ¿por qué se deberían utilizar mecanismos transformacionales, si su resultado puede ser generado directamente en la base? Esta pregunta no es un sinsentido, puesto que desde 1967 se desató el debate entre la hipótesis transformacional y la lexicista (cfr. Chomsky, 1970); precisamente, ésta, que acabaría por imponerse, propugnaba descargar a la gramática de buena parte de mecanismos transformacionales, generando en la base ciertos elementos. 
Por ello, mediante la RCE se plantea una paradoja: tal restricción se formula como un medio de limitar las cada vez más largas derivaciones transformacionales. Pero ya que pretende reducir las transformaciones comprobando si sus resultados coinciden con los obtenidos por las reglas sintagmáticas, la diferencia entre ambos tipos de reglas se oscurece, porque éstas se hacen demasiado relevantes para las transformaciones. En otras palabras, la RCE es un estímulo para restringir la gramática eliminando un nivel de representación. Pero (y aquí reside la paradoja), al tiempo, la RCE sólo se puede formular como control sobre el nivel a cuya eliminación parece apuntar. Notemos que es más simple una gramática con sólo el nivel sintagmático; si las transformaciones son necesarias (aspecto diáfano en GGT), es necesario justificar que efectivamente no llega con reglas de la base; pero la RCE hace lo contrario, al dejar toda la responsabilidad de legitimación de las transformaciones a la base.

4.3. Un tercer problema derivado de la RCE está relacionado con los elementos ' $\triangle$ ', los nudos vacíos postulados para asegurar que la estructura se mantiene constante, y por ello, se preserva. La exposición del tratamiento de las dos operaciones de pasivización muestra que esos elementos vacíos se introducen en la base para soportar el peso de ciertas operaciones que serán efectuadas posteriormente, en el curso de la derivación hacia la estructura superficial. Teniendo esa única justificación, es decir, no habiendo razones independientes que justifiquen la necesidad de su presencia en la estructura subyacente, tales nudos silentes son en buena medida estipulaciones ad hoc. No se busca legitimarlos de forma autónoma, al contrario de lo que sucedía con las transformaciones en relación a las reglas sintagmáticas. Por ello, aunque aseguran la estructura, se postulan con el fin de asegurar un mismo número de posiciones. En realidad, este mismo carácter ad hoc se puede percibir en el tratamiento mediante el que Emonds pretende resolver el problema de la opcionalidad de la FAgente (cfr. nota 15), puesto que la genera en la base para asegurar su presencia en etapas posteriores de la derivación; de otro modo, no se preservaría la estructura, dado que habría que introducir ilegítimamente una proyección no existente antes.

4.4. El último aspecto que deseo tratar es quizás el primordial: el hecho de que no todas las transformaciones sean preservadoras de la estructura, porque dos de las tres operaciones de la tipología no lo son. Por supuesto, la primera implicación que se deduce de este aspecto está muy relacionada con lo enunciado en 4.2.: en ese subapartado se afirmó que la RCE no podía limitar el exceso de poder a la hora de limitar la posibilidad de ofrecer diversas descripciones estructurales sobre los mismos hechos, esto es, limitar la capacidad generativa fuerte.

Dejando de lado este objetivo inalcanzable, centrémonos en la capacidad generativa débil: aunque la RCE la limita a sus cauces correctos, esta corrección está restringida sólo a las transformaciones preservadoras de la estructura, pero no a los otros dos tipos, locales y radicales. En los dominios de actuación de estas reglas, por tanto, al exceso de capacidad generativa fuerte se suma también el excesivo poder en generación débil, con lo que en esas dos clases de reglas ni siquiera se puede obtener ese 
resultado. La conclusión es obvia: las gramáticas ofrecidas por el modelo no se libran del exceso de capacidad generativa, de la sobregeneración. De nada sirve que se acoten aspectos de ciertos tipos de reglas si existen otras que no entran en tal limitación, puesto que el modelo seguirá sobregenerando.

Además, la preservación estructural de Emonds no puede desechar las diferentes operaciones transformacionales, como elisiones, supresiones y sustituciones, debido al carácter no generalizado de la RCE: mientras en las reglas que se someten a ella no se permite más que un desplazamiento, en las reglas locales y radicales siguen persistiendo esas operaciones. Por tanto, la existencia de la tipología transformacional provoca problemas en sí misma, debido a las diferencias entre unas operaciones y otras.

Los expuestos son, a mi juicio, los principales problemas que presenta la RCE, los cuales no menoscaban de ningún modo, como ya he recalcado, la enorme importancia teórica de la que en esencia goza la propuesta de Emonds: es la primera que intenta abordar la raíz del excesivo poder transformacional, constituida por las diferentes operaciones que estas reglas podían efectuar. Y sobre todo, porque en realidad desde su formulación, la RCE no fue abandonada en ningún momento, sino que se ha ido extendiendo y generalizando poco a poco, hasta eliminar las operaciones donde no se produzca un movimiento: elisión, sustitución e inserción. En el siguiente apartado se ofrecerá una breve panorámica de la extensión de la RCE, hasta llegar a ser una de las condiciones más importantes de la gramática17.

\section{Generalización del requisito de conservación estructural.}

En esta sección esbozaré cómo se ha extendido la noción de preservación estructural, hasta llegar a constituir uno de los principios que más han limitado el poder expresivo del modelo. Pero, a diferencia de la RCE, postulada específicamente en relación a las reglas transformacionales, la preservación estructural generalizada no se limita a esas reglas, sino que ha ampliado su alcance, de modo que todos los componentes gramaticales se ven afectados por ella, posibilitando así una limitación de opciones expresivas muy fuerte. Por ello, esta noción se muestra crucial en el modelo GB.

La extensión de la conservación estructural está inevitablemente unida al poder cada vez mayor del componente léxico, que pasó de ser mero componente pasivo por ejemplo en Chomsky (1965), simple lista de términos, a un componente realmente activo en GB. Para entender esta progresiva evolución es necesario prestar atención al debate, ya mencionado, entre la hipótesis lexicista y la transformacional, esto es,

17. Una buena muestra de la vigencia de la RCE de Emonds, si bien adaptada a los desarrollos más recientes de la teoría, se puede encontrar en Chomsky (1994: 17 y ss.), en donde se plantea cómo se puede adecuar la preservación estructural a un enfoque que prescinda de reglas sintagmáticas. 
entre generar directamente en el componente léxico o recurrir a las reglas transformacionales.

El debate en cuestión, originado en relación a ciertos derivados nominales (cfr. Chomsky 1970), fue ganado por el lexicismo. Esta victoria, no radical sino progresiva, marca el comienzo del auge del léxico, e igualmente, dispone de una gran importancia en relación al dominio transformatorio. El poder de las reglas de este dominio era tan fuerte que, por ejemplo tomando la operación de sustitución, mediante ella no sólo se podía cambiar un elemento perteneciente a la clase categorial X por otro también integrante de la misma clase (como sustitución de FN por un pronombre), sino que una regla podía variar incluso el estatus categorial de los dos elementos implicados en la permutación. Según Lees (1960), enfoque transformacional clásico en relación a los derivados nominales, una regla puede derivar una FN a partir de V. Así, se daba cuenta transformacionalmente de la relación entre:

(5) They claimed $\mathrm{O}$ [that John destroyed the city]

(6) They claimed $\mathrm{FN}$ [the destruction of the city]

Con el enfoque postulado en Chomsky (1970) esto empieza a variar, puesto que operaciones como la mencionada en este ejemplo son vetadas; en este sentido, Jackendoff (1972:13) constituye el destierro definitivo de esos mecanismos, al prohibirse que la regla actúe sobre el estatus categorial de los constituyentes. V y FN deben generarse en la base directamente.

Es bien cierto que el triunfo de la corriente lexicista no supuso una reducción drástica de las reglas, pero no lo es menos que desde entonces, tal reducción fue paulatina, y sobre todo, empezó a ser imparable, y cada vez más sistemática. Por ello, la prohibición de efectuar una operación como la efectuada en (5) y (6) no es aislada. De modo gradual, se empiezan a estipular muchas reglas transformatorias aisladas como innecesarias, en favor de la generación directa de los elementos implicados en el componente léxico. Por citar unos pocos ejemplos más, a partir de Bresnan (1970) los complementantes, introducidos hasta aquel entonces mediante reglas, disponen de una posición específica en la estructura profunda, Comp (que puede estar llena o vacía); esto significa que esos elementos son estipulados directamente en el léxico, al igual que los restantes mediante los que se conforman los niveles representacionales subyacentes $^{18}$. La generación de las secuencias relativas es otro buen ejemplo de la importancia creciente del léxico, puesto que en etapas anteriores se debían efectuar varias

18. La introducción transformacional del complementante sería incompatible con la prohibición de que una regla variara el estatus categorial de un elemento o segmento, puesto que, situándonos en el marco propuesto por Bresnan, una transformación de ese tipo variaría las propiedades subcategorizadoras de un segmento: una proyección O se convertiría mediante la inserción de 'that' en una proyección O-barra. Por ello, había razones independientes para vetar la solución transformacional. 
operaciones, tales como la inserción de la partícula relativa, eliminación de la FN sujeto u objeto que coincidiera referencialmente con la partícula, etc.; de nuevo, esto es variado, porque a partir de ese momento la partícula relativa aparece ya en la estructura profunda, lo que significa que el léxico accede a un dominio hasta entonces prohibido. Un tercer caso claro son los pronombres: el análisis clásico los insertaba mediante reglas, pero desde Dougherty (1969) se asume que deben insertarse directamente en el nivel subyacente; de nuevo, el léxico accede a elementos hasta entonces inaccesibles.

Si bien cada una de estas diferencias de tratamiento no es sino un leve arañazo al poder transformacional, tomadas en conjunto fueron reduciendo cada vez más tal poder, en beneficio del léxico. Lo que he tratado en el párrafo anterior se puede globalizar en un aspecto: la preservación de la estructura, esto es, la eliminación de aquellas reglas que no comportan un movimiento, puesto que de este modo no habría que variar el número de posiciones en el transcurso de la derivación transformacional. Así, cada vez se fue limitando la gran alteración a la que en el modelo clásico podía verse sometida la estructura profunda.

Esta evolución acabó en el auge absoluto del léxico, hasta el punto de que en GB la sintaxis es una proyección de tal componente; las secuencias son generadas gracias a que los elementos léxicos y sus rasgos temático-categoriales se trasvasan a la sintaxis (por la sintaxis X-barra y la teoría temática). Esto es formalizado por el Principio de Proyección (PP), que precisamente, se encarga de vigilar el trasvase; para Chomsky (1981:29), "Representations at each syntactic level (i.e., LF, and D- and S-structure) are projected from the lexicon in that they observe the subcategorization properties of lexical items."

De este modo, las propiedades del léxico deben ser trasvasadas a todos los niveles representativos de modo uniforme; y si tal trasvase es uniforme, debe ser preservador de la estructura, puesto que en el PP se exige que no se produzcan cambios de ningún tipo, independientemente del nivel representacional: si un elemento X está presente en el nivel $\mathrm{N}$, deberá reflejarse también en los restantes.

Lo interesante de este requisito en relación a las transformaciones consiste en que automáticamente no tienen cabida la mayor parte de operaciones que estas reglas podían hacer, porque sustitución, inserción y elisión atentarían contra la condición establecida por el PP, ya que variarían posiciones y/o rasgos subcategorizadores.

En cuanto al cuarto tipo, el movimiento, en principio es el único posible, satisfaciendo el requisito tan estricto al que el PP obliga; frente a los otros tres, la aplicación de la regla no varía el número de elementos, aunque uno cambie de posición. Sin embargo, dejando así las cosas, la estructura resultaría alterada:

1. Si un elemento se desplaza a una posición no estipulada por las reglas sintagmáticas (como sucedía en el marco de Chomsky 1965), la nueva posición debe ser creada, mientras que la posición originaria se perdería, alterando las propiedades de las entradas léxicas. 
2. Si optamos por asumir que el mencionado elemento ocupa una posición previamente existente, creada por el componente sintagmático (como en el movimiento de palabras Qu-, que ocupan las posiciones Comp jerárquicamente superiores), aunque la posición receptora existiría, la primitiva se perdería, supuesto que violaría de nuevo el PP.

En cualquiera de las dos situaciones, el lugar desde el que se produce el desplazamiento se pierde, y aunque el elemento movido no desaparece, sino que cambia de posición, las características mediante las que esa pieza era reclamada por otra ya no están presentes. Por ejemplo, si una FN objeto de un V se desplaza, las características temático-categoriales mediante las que $\mathrm{V}$ selecciona la FN no estarían ya presentes junto a $\mathrm{V}$, quebrantándose el trasvase uniforme del léxico a la sintaxis, por una pérdida de estructura ${ }^{19}$.

En otras palabras, un movimiento estará legitimado sólo si deja una marca en el lugar desde el que se originó tal desplazamiento. De este modo, se mantiene la posición primitiva de la pieza movida, asegurando que la información sea la misma en todos y cada uno de los niveles representativos, o dicho de otra manera, se asegura la estricta correspondencia entre la información presente en la estructura-P y la de los restantes niveles. Esta exigencia es satisfecha por medio de la huella, elemento vacío de contenido aunque relevante sintáctica e interpretativamente: cuando se produce un desplazamiento, se genera una huella en la posición primitiva, preservando así la estructura, ya que se asegura la no eliminación de tal posición.

Tras esta breve visión, tenemos ya la forma en la que se pudo extender la preservación estructural de Emonds, haciéndola muy fructífera para la teoría de la gramática. Se puede intuir ya la forma en la que los problemas presentados en relación a la RCE fueron superados:

1. La restricción de Emonds no podía limitar la formulación de descripciones alternativas sobre los mismos hechos, con lo que no conseguía restringir la capacidad generativa fuerte del modelo. Sin embargo, mediante el enfoque que se deriva del PP, sí se ha restringido espectacularmente el poder generativo del modelo, gracias a la gran limitación de las reglas: la única operación que puede efectuar una transformación es un movimiento, eliminando así la gama operacional de estas reglas.

2. Una pregunta derivada del enfoque de Emonds consistía en cuestionar la necesidad de las transformaciones si sus resultados se justifican mediante las reglas de la base. Tras la evolución señalada, esa pregunta no tiene ya sentido: la transformación es concebida como un último recurso, una operación que el léxico por sí mismo no puede efectuar.

3. Igualmente se señalaba que las posiciones ' $\triangle$ ' eran claramente ad hoc, porque su única razón de ser provenía de la necesidad de recurrir a ellas posteriormente,

19. La cual, así pues, no debe ser entendida sólo como una pérdida de posiciones o elementos, sino también de características de los mismos. 
durante la derivación transformacional. En general, las categorías vacías constituían un sistema inacotado, adaptado a las necesidades concretas de uso (cfr. nota 13). Sin embargo, las categorías vacías exigidas por el PP constituyen un sistema muy reducido, y limitado a aparecer bajo condiciones muy severas. Además, siempre que se produce un movimiento, éste deja una huella, con lo que no se debe estipular para cada operación concreta si es necesario dejar tal marca.

4. Quizás el mayor problema de la propuesta de Emonds consistía en su carácter no general: algunas reglas eran preservadoras de la estructura, pero otras no. Sin embargo, en el enfoque GB esta asimetría se elimina de modo sencillo: toda regla debe preservar la estructura, por lo que la tipología propuesta por Emonds desaparece, con el consiguiente beneficio tanto para la limitación de los mecanismos gramaticales como para el correlato psicológico antes tratado.

\section{A modo de conclusión.}

En el último párrafo he mencionado que toda transformación debe preservar la estructura. Sin embargo, esta exigencia no remata en las reglas de movimiento, sino que es necesario que todos los mecanismos, componente y niveles de la gramática preserven la estructura. Precisamente, es éste el cambio más acusado entre la RCE y la preservación estructural generalizada: se puede afirmar que el PP consiste en la extensión de la RCE de Emonds a todos los dominios de la gramática, de modo que se limiten todos los mecanismos expresivos, y no sólo el transformacional.

Este autor buscaba medios para restringir las historias transformacionales, al menos de modo parcial, y para ello formula unas reglas que no pueden alterar la estructura; el componente sintagmático justifica el resultado de las transformaciones, asegurando la preservación. El PP responde al mismo deseo, pero desde una perspectiva diferente, mucho más abarcadora y extendida, puesto que la preservación estructural se sitúa, gracias al PP, en el comienzo del proceso, en el componente léxico. Dado que la sintaxis es una proyección del léxico, y debiendo ser las piezas y sus características uniformemente mantenidas en todos los niveles, no es necesario estipular de forma independiente la necesidad de preservar la estructura en cada nivel o componente aisladamente. De ahí que, gracias al PP, la estructura inicial debe ser preservada en cualquier situación. La restricción que Emonds vinculaba directamente a un tipo de reglas puede así ser derivada de principios independientes a ellas.

Para concluir, gracias al poder cada vez más intenso del componente léxico, las transformaciones han ido perdiendo poder expresivo, algo muy necesario para restringir las opciones que unos mecanismos tan costosos como las reglas ofrecían (en un modelo íntegramente basado en ellas). Sin embargo, esta disminución del poder expresivo (mecanismos expresivos) no significa pérdida para el modelo de adecuación descriptiva, y sí ganancia de la explicativa. De este modo, la preservación extendida 
VÍCTOR M. LONGA

de la estructura ha ayudado en gran medida a resolver la tensión entre descripción y explicación.

\section{Bibliografía}

BACH, E. (1962), "The Order of Elements in a Transformational Grammar of German", Language, 38, 263-69.

BERWICK, R. \& A. WEINBERG (1986), The Grammatical Basis of Linguistic Performance, Cambridge, MA, MIT Press.

BRESNAN, J. (1970), "On Complementizers: Toward a Syntactic Theory of Complement Types", Foundations of Language, 6, 297-321.

CHOMSKY, N. (1964), "The Logical Basis of Linguistic Theory”, en Lunt, H., ed., Proceedings of the Ninth International Congress of Linguists, The Hague, Mouton, 914-77.

CHOMSKY, N.A. (1965), Aspects of the Theory of Syntax, Cambridge, MA, MIT Press.

CHOMSKY, N. (1970), "Remarks on Nominalizations”, en Jacobs, R. \& P. Rosenbaum, eds., Readings in English Transformational Grammar, Waltham, MA, Gin \& Co., 184-221.

CHOMSKY, N. (1972), "Some Empirical Issues in the Theory of Transformational Grammar”, en Peters, S., ed., Goals of Linguistic Theory, New Jersey, PrenticeHall, 63-130.

CHOMSKY, N. (1973), “Conditions on Transformations”, en Anderson, S. \& P. Kiparsky, eds., A Festschrift for Morris Halle, New York, Holt, Rinehart \& Winston, 232-286.

CHOMSKY, N.A. (1976), "Conditions on Rules of Grammar", Linguistic Analysis, 2,4, 303-51.

CHOMSKY, N.A. (1981), Lectures on Government and Binding, Dordrecht, Foris.

CHOMSKY, N.A. (1986), Knowledge of Language, New York, Praeguer Publishers.

CHOMSKY, N.A. (1994), "Bare Phrase Structure”, MIT Occasional Papers in Linguistics, 5.

D'INTRONO, F. (1979), Sintaxis transformacional del español, Madrid, Cátedra.

D'INTRONO, F. (1985), Sintaxis y semántica en la lingüística generativa: el modelo gramatical GB, Caracas, Centro de Investigaciones "Andrés Bello".

DOUGHERTY, R. (1969), “An Interpretative Theory of Pronominal Reference”, Foundations of Language, 5, 488-519.

EMONDS, J. (1970), Root and Structure-Preserving Transformations, MIT Ph.D. Dissertation.

EMONDS, J. (1976), A Transformational Approach to English Syntax, New York, Academic Press. 
JACKENDOFF, R. (1972), Semantic Interpretation in Generative Grammar, Cambridge, MA, MIT Press.

KATZ, J. \& P. POSTAL (1964), An Integrated Theory of Linguistic Descriptions, Cambridge, MA, MIT Press.

KIMBALL, J. (1972), “Cyclic and Linear Grammars”, en Kimball, J., ed., Syntax and Semantics, 1, New York, Seminar Press, 63-80.

KIMBALL, J. (1973), The Formal Theory of Grammar, New Jersey, Prentice-Hall.

LEES, R. (1960), The Grammar of English Nominalizations, The Hague, Mouton.

NEWMEYER, F. (1980), Linguistic Theory in America, New York, Academic Press.

PETERS, S. \& R.W. RITCHIE (1971), "On Restricting the Base Component of Transformational Grammars", Information and Control, 18, 483-501.

PETERS, S. \& R.W. RITCHIE (1973), “On the Generative Power of Transformational Grammars", Information Sciences, 6, 49-83.

POSTAL, P. (1970), “On Coreferential Complement Subject Deletion”, Linguistic Inquiry, 1, 439-500.

ROSS, J. (1967), Constraints on Variables in Syntax, MIT Ph.D. Dissertation.

ROSS, J. (1969), “Guess Who?”, Papers from the Fifth Regional Meeting of the CLS, 252-86.

ROSS, J. (1970), "Gapping and the order of constituents", en Bierwisch, M. \& K. Heidolph, eds., Progress in Linguistics, The Hague, Mouton, 249-259

WASOW, T. (1985), "Postscript" a Sells, P. (1985), Lectures on Contemporary Syntactic Theories, Leland Stanford Junior University, 193-205.

WEINBERG, A. (1988), "Mathematical properties of grammars", en Newmeyer, F., ed., Linguistics: The Cambridge Survey. I. Linguistic Theory: Foundations, Cambridge, Cambridge University Press, 416-29. 